\title{
Características que influyen en la susceptibilidad a la enfermedad periodontal en la población pediátrica. Revisión integradora*
}

\author{
Characteristics Influencing Susceptibility to Periodontal Disease in Pediatric Population. \\ Integrative Review
}

Características que influenciam a suscetibilidade à doença periodontal na população

pediátrica. Revisão integrativa

Fecha de recepción: 19-02-2020 | Fecha de aceptación: 01/07/2020

\author{
MARio RAFAel Romero Sánchez \\ Pontificia Universidad Javeriana, Bogotá, Colombia. \\ romero.mario@javeriana.edu.co; https://orcid.org/0000-0003-0927-2371
}

\author{
María Marcela Colmenares Millán \\ Pontificia Universidad Javeriana, Bogotá, Colombia. \\ colmenaresm@javeriana.edu.co; https://orcid.org/0000-0002-3893-6651
}

Correspondencia: romero.mario@javeriana.edu.co; colmenaresm@javeriana.edu.co

*Investigación original 


\section{doi: https://doi.org/10.11144/Javeriana.uo39.cise}

Cómo citar: Romero Sánchez MF, Colmenares Millán MM. Características que influyen en la susceptibilidad a la enfermedad periodontal en la población pediátrica. Revisión integradora. Univ Odontol. 2020; 39. https://doi.org/10.11144/Javeriana.uo39.cise

\section{RESUMEN}

Antecedentes: Durante la niñez y la adolescencia ocurren numerosos cambios biológicos que deben considerarse por el impacto que tienen en estados de salud o enfermedad periodontal. Estos cambios se relacionan con las propiedades histomorfológicas, la composición de la biopelícula y el desarrollo gradual del sistema inmune durante las diferentes etapas de crecimiento y desarrollo. Este es un tema ampliamente expuesto en población adulta pero poco descrito en la población pediátrica. Objetivo: Sintetizar la evidencia actual sobre las características que influyen en la susceptibilidad a la enfermedad periodontal en la población pediátrica. Métodos: Se realizó una revisión integradora de artículos, estados del arte y textos completos y con base en la experiencia clínica. Se tuvieron en cuenta las características morfológicas, microbiológicas e inmunológicas del periodonto. Resultados: En los tejidos periodontales de los niños existe mayor grosor y número de capas del epitelio de unión, infiltrado celular inflamatorio adyacente al epitelio de unión, mayor cantidad de vasos y predominio de fibras del tejido conectivo laxas. Se presenta un incremento de patógenos periodontales a medida que aumenta la edad y es proporcional al grado de inflamación que se encuentra asociado a un sistema inmune inmaduro. Conclusión: El riesgo de desarrollar gingivitis a temprana edad es bajo. Sin embargo, a medida que el niño crece, aumenta su 
susceptibilidad a la inflamación gingival mediada por las características de la microbiota y factores relacionados con la edad como el desarrollo y la madurez del sistema inmune.

\section{Palabras clave}

biopelícula; enfermedad periodontal; histomorfología oral; histomorfología periodontal; inmunología oral; inmunología periodontal; microbiología oral; microbiología periodontal; odontología; odontopediatría; periodoncia; periodonto en niños; salud periodontal

\section{ABSTRACT}

Background: During childhood and adolescence, numerous occurring biological changes must be considered due to their impact on health or periodontal disease conditions. These changes are related to histomorphological properties, the composition of the biofilm, and the gradual development of the immune system during the different stages of growth and development. This is a subject that is widely exposed in adults, but little described in pediatric population. Purpose: To synthesize the current evidence on the characteristics that influence the susceptibility to periodontal disease in the pediatric population. Methods: An integrative review of articles, state of the art and full texts was carried out, based on clinical experience. Morphological, microbiological, and immunological characteristics of the periodontium were taken into account. Results: In the periodontal tissues of children there are more and thicker layers of the junctional epithelium, inflammatory cell infiltrate adjacent to the junctional epithelium, greater numbers of vessels, and a predominance of loose connective tissue fibers. Periodontal pathogens grow as age increases, which is proportional to the degree of inflammation that is associated with an immature immune system. Conclusion: The risk 
of developing gingivitis at an early age is low. However, as the child grows, their susceptibility to gingival inflammation, mediated by characteristics of the microbiota and age-related factors such as the development and maturity of the immune system, increases.

\section{Keywords}

biofilm; dentistry; pediatric dentistry; pediatric periodontics; periodontal disease; periodontal histomorphology; periodontal immunology; periodontal microbiology; periodontium in child; periodontal health

\section{RESUMO}

Antecedentes: Durante a infância e a adolescência ocorrem inúmeras alterações biológicas que devem ser consideradas devido ao impacto que têm nos estados de saúde ou doença periodontal. Essas alterações estão relacionadas às propriedades histomorfológicas, à composição do biofilme e ao desenvolvimento gradual do sistema imunológico durante as diferentes fases de crescimento e desenvolvimento. Esse é um assunto amplamente exposto na população adulta, mas pouco descrito na população pediátrica. Objetivo: Sintetizar as evidências atuais sobre as características que influenciam a suscetibilidade à doença periodontal na população pediátrica. Métodos: Foi realizada uma revisão integrativa de artigos, estado da arte e textos completos, com base na experiência clínica. As características morfológicas, microbiológicas e imunológicas do periodonto foram levadas em consideração. Resultados: Nos tecidos periodontais de crianças há maior espessura e número de camadas do epitélio juncional, infiltrado de células inflamatórias adjacentes ao epitélio juncional, maior número de vasos e predomínio de fibras de tecido conjuntivo frouxas. Há um aumento dos 
patógenos periodontais com o aumento da idade e é proporcional ao grau de inflamação que está associado a um sistema imunológico imaturo. Conclusão: O risco de desenvolver gengivite em idade precoce é baixo. Porém, à medida que a criança cresce, aumenta sua suscetibilidade à inflamação gengival, mediada por características da microbiota e por fatores relacionados à idade, como o desenvolvimento e a maturidade do sistema imunológico.

\section{Palavras Chave}

biofilme; doença periodontal; histomorfologia oral; histomorfologia periodontal; imunologia oral; imunologia periodontal; microbiologia oral; microbiologia periodontal; odontologia; odontopediatria; periodontia; periodonto em criança; saúde periodontal

\section{INTRODUCCIÓN}

La salud periodontal es un estado libre de enfermedad periodontal inflamatoria, la cual se puede evaluar histológica y clínicamente, y así considerarla desde un contexto preventivo hasta uno terapéutico. Las enfermedades periodontales deben entenderse como enfermedades complejas de naturaleza multifactorial que comprenden la interacción de biopelícula subgingival y la respuesta inmune e inflamatoria, así como factores modificantes del medio ambiente. Por lo tanto, pueden agruparse en tres categorías principales: microbiológicas, del huésped y del medio ambiente (1).

Estudios longitudinales y transversales indican que la severidad y prevalencia de la enfermedad periodontal incrementan gradualmente desde la infancia hasta la adultez, debido a una reactividad que depende de la edad a la biopelícula. Con similares cantidades de biopelícula, los niños 
presentan menor gingivitis severa que individuos mayores (2-4). En este contexto, la interrelación entre biopelícula, inflamación gingival y profundidad del surco alrededor de los dientes ha recibido especial atención: si la inflamación gingival en individuos jóvenes presagia enfermedad periodontal en el curso del tiempo (5). Sin embargo, existe menor evidencia, en estudios prospectivos longitudinales, que los signos de inflamación periodontal en la niñez vayan a predecir un fuerte riesgo de enfermedad periodontal en la adultez (6). Aunque se ha sugerido que esta tendencia puede estar relacionada con diferencias en la cantidad de biopelícula acumulada (7), resultados obtenidos bajo cantidades comparables de biopelícula en diferentes edades indican que otros factores están involucrados.

Por lo tanto, las diferencias en las manifestaciones clínicas de la enfermedad periodontal que se incrementan con la edad son atribuidas a los cambios funcionales de las estructuras periodontales, al establecimiento y maduración de la microflora oral y al desarrollo gradual del sistema inmune (8-10). Las características anteriormente mencionadas motivaron a realizar una búsqueda integradora exhaustiva de las diferencias presentadas en la población pediátrica.

\section{MATERIALES Y MÉTODOS}

Con base en la experiencia clínica, investigativa y docente, durante las últimas dos décadas se ha compilado literatura representada en artículos, estados del arte y textos completos. Con ellos se realizó una revisión integradora de este tema que está poco descrito en la población pediátrica. Se tuvieron en cuenta las características morfológicas, microbiológicas e inmunológicas del periodonto. 


\section{RESULTADOS}

\section{Características morfológicas}

Se han descrito las diferencias morfológicas entre los tejidos gingivales clínicamente sanos de niños con dentición primaria y adultos. Bimstein (11), en un artículo publicado en 1987 sobre las consideraciones periodontales en el paciente niño, describe que la apariencia y morfología de los tejidos periodontales están sujetos a cambios continuos durante el crecimiento y desarrollo. Así, la unidad gingival en los niños presenta características diferenciales en color, punteado gingival, profundidad del surco gingival, amplitud de la encía adherida y apariencia radiográfica.

Teniendo en cuenta lo anterior, los tejidos gingivales en la población pediátrica difieren de los de los adultos en su aspecto clínico y en la resistencia a la aparición de la enfermedad periodontal. Delaney (12), en su capítulo sobre anormalidades periodontales y de tejidos blandos, describe que la encía clínicamente sana en el preescolar suele ser más rojiza, por una mayor vascularización; tiene un epitelio más delgado, menos queratinizado y más translúcido. La superficie gingival puede ser lisa o levemente moteada y los tejidos pueden mostrar una consistencia más firme y elástica. El contorno de la encía marginal muestra una forma redondeada o enrollada, por la constricción cervical que tienen los dientes primarios $(10,11)$.

La profundidad del surco alrededor de los dientes temporales es de 2,5 $\mathrm{mm}$ en promedio, al sondeo, y en los sitios vestibular y lingual es menor que la de los proximales. Durante el recambio dental, la profundidad del surco gingival tiende a aumentar en los dientes deciduos en la medida en que se produce la reabsorción radicular fisiológica y al erupcionar los dientes permanentes. Al mismo 
tiempo que aumenta la dimensión de la corona clínica, el surco gingival disminuye. La profundidad del surco en los dientes recién erupcionados se debe a la presencia de una inserción primaria delicada que ofrece menor resistencia al sondaje, a la altura relativa de la unión amelocementaria con respecto a la cresta ósea y a la gingivitis por la erupción (10). El ancho de la encía es mayor en el maxilar superior que en el inferior, parámetro que aumenta en la transición de los dientes primarios a los permanentes. El espacio interdental en el niño de corta edad está determinado por elementos como el tamaño, la forma y el espacio entre los dientes deciduos con papilas aplanadas y redondeadas (11) (tabla 1).

TABLA 1

CARACTERÍSTICAS MORFOLÓGICAS DEL TEJIDO GINGIVAL EN NIÑOS

\begin{tabular}{ll} 
Característica & \multicolumn{1}{c}{ Niños } \\
\hline Color & Rojizo \\
Aspecto & Liso brillante \\
Textura & Punteado en cascara de naranja menos pronunciado \\
Consistencia & Flácida y retráctil \\
Margen gingival & Redondeado gingival \\
Profundidad del surco & $\begin{array}{l}\text { 2.5mm. Depende del proceso fisiológico de } \\
\text { erupción y exfoliación dental (variable según etapa) }\end{array}$ \\
Papilas & Aplanadas y redondeadas \\
\hline
\end{tabular}

El análisis radiográfico es un componente crítico para determinar el estado clínico del periodonto, el cual debe comprender la presencia de lámina dura continua, la ausencia de pérdida ósea en las furcaciones y la distancia promedio de $2 \mathrm{~mm}$ desde la cresta ósea hasta la unión amelocementaria. En este aspecto se presentan variaciones que depende de la edad del paciente, el tipo de diente, la angulación de los dientes y la presencia de atrición (1).

El alvéolo que rodea los dientes deciduos muestra menos trabéculas, mayores espacios medulares y menor calcificación. El ligamento periodontal es más amplio que en los adultos (11). Con la edad, el hueso se vuelve más denso. Debido a la discrepancia entre el nivel de la 
unión amelocementaria de los dientes deciduos y el de los permanentes en erupción, el curso de la cresta ósea puede dar la impresión de defectos óseos angulares (10) (tabla 2).

TABLA 2

CARACTERÍSTICAS RADIOGRÁFICAS EN NIÑOS

\begin{tabular}{ll}
\hline \multicolumn{1}{c}{ Características } & \multicolumn{1}{c}{ Niños } \\
\hline Cemento radicular & Más delgado, menos denso \\
Ligamento periodontal & $\begin{array}{l}\text { Espacio más amplio, tejido menos denso, más vascular y } \\
\text { celular }\end{array}$ \\
\hline $\begin{array}{l}\text { Espacios medulares amplios, menor trabeculado, lámina } \\
\text { dura delgada, crestas óseas planas, menor grado de } \\
\text { calcificación, mayor vascularización }\end{array}$ \\
\hline
\end{tabular}

Bimstein y Matsson (9), con base en investigaciones clínicas e histológicas de las diferencias morfológicas del tejido gingival de las denticiones primaria y permanente, observaron que el infiltrado celular inflamatorio en la permanente se encuentra en la porción coronal de la encía libre, mientras que, en la dentición decidua, la lesión inflamatoria ocupa una porción de tejido más angosta a lo largo del epitelio de unión. Adicionalmente, el epitelio de unión en la encía de los dientes deciduos es más grueso que en los permanentes, lo que puede ocasionar una reducción en la permeabilidad de las estructuras a las toxinas bacterianas en la encía.

Zappler también describió acerca de la dentición primaria un estrato córneo y un epitelio oral más delgado y menos queratinizado, mayor vascularización en el tejido conectivo gingival y un patrón menos organizado de fibras colágenas (13). Estos hallazgos fueron controvertidos por Matsson y Bimstein al observar las características histológicas de la encía asociada a dientes deciduos y permanentes. Encontraron un número más alto de leucocitos en el tejido conectivo adyacente a la zona más apical del epitelio de unión y fibras colágenas menos orientadas y organizadas, así como mayor densidad de las fibras colágenas en el tejido conectivo subyacente al epitelio oral del periodonto en los niños. Detectaron también migración apical del epitelio de unión en la superficie 
radicular de los dientes deciduos debido al proceso de recambio dental. Asimismo, no confirmaron una mayor vascularidad o menores patrones de haces de fibras colágenas bien diferenciadas. Tampoco fue mayor la densidad de fibras colágenas en la encía de dientes deciduos, ni hallaron una menor capa de epitelio oral y menor queratinización $(4,14)$.

La severidad y 1 prevalencia de la enfermedad periodontal aumenta gradualmente de la infancia a la adultez, aumento que se puede atribuir al acúmulo de biopelícula. En niños, la enfermedad periodontal es frecuentemente descrita como limitada a gingivitis en el margen del tejido blando, donde formas más avanzadas de la enfermedad son características de la edad adulta. La inflamación gingival en niños es más leve que en adultos jóvenes o niños de mayor edad con cantidades similares de biopelícula. (2)

Desde 1973, diferentes autores han descrito una baja severidad de la gingivitis encontrada en niños y una prevalencia entre $18 \%$ y $74 \%$ en niños de 6 años, que aumenta con la edad y llega a un 80 $\%$ en pacientes entre 11 y 13 años. Disminuye al terminar la dentición mixta (15). Arango et al. (16) observaron que la presencia de gingivitis aumenta con la edad y una prevalencia entre 18,78 $\%$ y $56,25 \%$ en 128 niños entre 5 y 8 años. Tal gingivitis mostraba un incremento en el proceso de recambio dental. Por otra parte, Taboada y Talavera, en México, hallaron una prevalencia de gingivitis en el $39 \%$ de 77 niños preescolares entre 4 y 5 años (17), en tanto que Zaror et al. (18) describieron una prevalencia del $93,1 \%$ de gingivitis con una correlación positiva con caries y con el índice de placa bacteriana en niños de 4 años de edad en Chile. 
Benitez y Romero (19), en estudio realizado en 376 niños sistémicamente sanos, evaluaron la relación entre la edad y la presencia de gingivitis inducida por biopelícula en niños de 3 a 12 años por medio del índice gingival y de placa bacteriana de Löe y Silness. Encontraron que durante períodos de la vida, como la niñez, el riesgo de desarrollar gingivitis es muy bajo, mientras que en otros, el tejido gingival es más susceptible a reaccionar con inflamación a la irritación causada por bacterias.

Baer y Benjamin en 1974 (20), Longhurst et al. en 1977 (21) y Walker y Mackensie en 1982 (22) afirman, con respecto a las características clínicas e histológicas en la encía de niños con gingivitis inducida por biopelícula, que la enfermedad periodontal en niños usualmente presenta un enrojecimiento e inflamación de la encía sin formación de bolsa periodontal. La ausencia de bolsa se ha atribuida a la escasa migración apical del epitelio de unión.

En un estudio realizado por Bimstein et al. (23) sobre gingivitis en la dentición decidua, se revela una migración apical del epitelio de unión en un 53 \% y que está acompañada por un alargamiento del mismo, con una migración apical de su parte coronal y una reducción en la profundidad del surco. Esto sugiere que en la dentición decidua, durante la migración apical del epitelio de unión, no hay formación de bolsa periodontal pero sí se presenta recesión gingival. Este fenómeno se ha relacionado con una combinación de factores como la erupción pasiva y el proceso de erupción y exfoliación de la dentición decidua. Además, el surco gingival fue poco profundo $(0,3 \pm 1,9 \mathrm{~mm})$ y el infiltrado celular inflamatorio estuvo presente en todos los sitios examinados.

Romero et al. en 2011 (24) publicaron un estudio sobre las diferencias histológicas entre la encía de niños y adultos jóvenes con gingivitis inducida por biopelícula. Informaron de un grosor del epitelio 
oral en la zona coronal significativamente mayor en niños que en adultos. El promedio de la profundidad del surco gingival fue de 2,4 \pm 0,68 mm en niños y 2,9 \pm 0,44 en adultos jóvenes. Estos hallazgos son mayores que los descritos sobre la encía clínicamente sana en dentición decidua por Bimstein en 1987 (11), quien encontró un promedio de 2,1 mm con un rango de 0,5 mm a 4,6 mm, y Rosenblum, citado por Jiménez, (25) con 2,1 $\mathrm{mm} \pm$ 0,2 $\mathrm{mm}$. Ello podría indicar que esta profundidad aumenta por la respuesta inflamatoria ante la presencia de biopelícula y los procesos fisiológicos. El grosor y el número de capas del epitelio de unión fueron mayores a la altura coronal en los adultos. Se encontró predominio de tejido conectivo laxo en los niños corroborando los hallazgos de estudios previos, al compararse con adultos jóvenes, en quienes fue más frecuente el tejido fibroso (70 \%). Este estudio mostró pocas diferencias histomorfológicas entre el tejido de dientes deciduos y permanentes con diagnóstico de gingivitis inducida por biopelícula. Los resultados indican que las diferencias estructurales pueden ser poco importantes una vez se establece la lesión inflamatoria (24).

Al realizar el examen clínico en un paciente pediátrico, también es importante identificar los cambios que ocurren en el periodonto en presencia de materiales de restauración como las coronas de acero. Se ha reportado que la gingivitis se presenta frecuentemente alrededor de los dientes deciduos restaurados con coronas de acero que causada por diversos factores, principalmente una inadecuada técnica durante todo el proceso terapéutico (26-28). Ercoli y Caton (29) mencionan el hallazgo de Schatzle acerca de la inflamación gingival en adultos, la que suele ser significativamente mayor alrededor de los márgenes de restauraciones subgingivales.

Madrigal et al. (30) llevaron a cabo una revisión sistemática sobre los factores predisponentes de la inflamación gingival asociada a coronas de acero en dientes temporales en población pediátrica. 
No encontraron evidencia científica suficiente para corroborar que la adaptación de las coronas de acero sea uno de los factores predisponentes de enfermedad gingival en el paciente pediátrico, ni que muestre la alteración del tejido periodontal por la invasión del espesor biológico debido a la sobrextensión de las coronas de acero. La presencia de excesos de material cementante no estaba ampliamente documentada. La evidencia analizada en dicha revisión no permite confirmar lo hallado por Myers en 1975 (28), quien observó una asociación significativa entre los defectos de la corona y la evidencia clínica de gingivitis. Entonces, la literatura no es contundente en relación con el comportamiento del tejido gingival de la población pediátrica. En 1983, Checchio et al. (31) argumentaron que los cambios en el tejido gingival se presentaban como respuesta a la inflamación inicial relacionada con la colocación de la corona. Tales cambios que se resolvían con el tiempo como respuesta al restablecimiento y adaptación del tejido.

El término enfermedad periodontal abarca todas las condiciones patológicas de los tejidos periodontales. Ella constituye la segunda patología bucodental más prevalente en el mundo (32). En el consenso establecido entre la Academia Estadounidense de Periodoncia y la Academia Europea de Periodoncia acerca de la periodontitis, Papapanou et al. (33) describen la periodontitis como una enfermedad inflamatoria crónica multifactorial asociada a biopelícula disbiótica y que se caracteriza por destruir progresivamente los tejidos de soporte periodontal. Es uno de los mayores problemas de salud pública debido a su alta prevalencia. Las formas de periodontitis se reorganizaron teniendo en cuenta la fisiopatología de las entidades en periodontitis, periodontitis necrosante y periodontitis como manifestación de enfermedad sistémica (33). 
La periodontitis puede ser la manifestación oral de una enfermedad sistémica o un desorden de inmunodeficiencia en la población pediátrica y adolescente. Albandar et al. en 2018 mencionan que la categorización de dicha periodontitis depende de la clasificación de la enfermedad primaria establecida en clasificación internacional de enfermedades de la Organización Mundial de la Salud (34). La enfermedad periodontal y algunos desordenes sistémicos comparten factores genéticos y ambientales que llevan a la manifestación de ambos tipos de condiciones. Varios desordenes afectan los mecanismos de inflamación gingival y que alteran la respuesta inmune ante la infección periodontal. Otros generan defectos en la encía o en el tejido conectivo que conducen a cambios metabólicos en el huésped y pueden afectar los tejidos periodontales o seguir otras vías. Varios factores del huésped se relacionan con la patogénesis periodontal. Se incluyen la respuesta inmune, factores anatómicos y factores estructurales en los tejidos. La mayoría está determinada por el perfil genético de la persona y pueden modificarse por factores ambientales y comportamentales. Por tanto, la pérdida de los tejidos de soporte periodontal es una manifestación común de algunos desordenes sistémicos relevantes en el diagnóstico e implicaciones terapéuticas (34).

\section{Características microbiológicas}

La cavidad oral es un ecosistema altamente diverso con más de 600 especies microbianas que colonizan diferentes microambientes. La colonización microbiana de la cavidad oral inicia desde el nacimiento durante el paso del recién nacido a través del canal vaginal. Al comienzo, la composición de microbiota es relativamente simple (35). Sin embargo, diferentes factores afectan el ecosistema, entre ellos, la erupción de los dientes deciduos y el recambio de la dentición temporal por la dentición permanente, que proporciona superficies y sitios de unión que favorecen la colonización de bacterias y afectan los tejidos dentales y periodontales (36). 
Existe poca información sobre el efecto de la erupción de los dientes deciduos sobre la adquisición de los microorganismos periodontales. Es posible que el surco gingival alrededor de los dientes deciduos no actúe como un medio ambiente conveniente, en términos de profundidad y anaerobiosis, para la colonización de microorganismos asociados a la enfermedad periodontal. Por otro lado, la respuesta inmune inmadura en el niño puede influenciar la composición de la microflora oral, cuya composición cambia a medida que el ser humano crece y madura (37).

Papaioannou et al. (2009) estudiaron la microbiota de diferentes superficies orales en niños saludables. Encontraron similares perfiles bacterianos en muestras de biopelícula subgingival y supragingival con altas proporciones de especies de Actinomyces y bacterias del complejo verde. Algunos patógenos periodontales del complejo rojo, como Tannerella forsythia, Porphyromonas gingivalis ( $P$. gingivalis) y Treponema denticola, se incrementaban con la edad (35). Gafan et al. (2004) hallaron, en un estudio realizado en 118 niños con y sin gingivitis, altas proporciones de $P$. gingivalis y Actinobacillus actinomycetemcomitans, así como la presencia de Tannerella forsythensis en pacientes sin gingivitis. Comprobaron así la presencia de patógenos periodontales en la biopelícula de niños con salud gingival clínica mantenida y con gingivitis (38).

Querubín et al. en 2018, en una revisión sistemática de la literatura realizada sobre la composición de la microbiota del surco gingival en población pediátrica con dentición temporal y mixta y su relación con el estado periodontal, puntualizan que a nivel del surco gingival existe gran variedad de microorganismos, la cual puede variar sustancialmente dependiendo de las modificaciones en el microambiente relacionadas con las características fisiológicas presentes en los niños y 
adolescentes. En dicha revisión, se encontró, que los perfiles microbianos de la biopelícula supra y subgingival en niños son similares en más de un $80 \%$ con predominio de Actynomices spp y Streptococcus spp, principalmente S. sanguinis (39).

Drummond et al. en 2017, referencian que Crielaard et al. en su estudio sobre la composición de la microflora en saliva de niños, jóvenes y adolescentes, encontraron un microbioma salivar complejo en niños de tres años, que cambió con la edad, siendo diferente en la pubertad o en la edad adulta. Además, fueron encontradas proporciones incrementadas de patógenos periodontales como bacteroides spp. y espiroquetas spp. en niños de mayor edad (40).

Durante la pubertad se llevan a cabo una serie de cambios importantes en los individuos donde se encuentra incrementada la secreción de hormonas esteroideas que pueden afectar el ecosistema subgingival. Estos cambios hormonales conllevan una variación en la composición de la biopelícula hacia una microflora patógena. Por lo tanto, en la pubertad hay un impacto hormonal sobre la inflamación gingival (40).

\section{Características inmunológicas}

Page, Genco et al., citados por Bimstein y Ebersole en 1989, refieren que los mecanismos por los cuales la biopelícula bacteriana inicia la enfermedad periodontal están presumiblemente basados, sobre el efecto de los productos metabólicos y antigénicos de los microorganismos sobre el tejido periodontal. Estos componentes causan un incremento de la permeabilidad del epitelio surcular, seguido por la penetración de los tejidos, provocando respuestas inflamatorias e inmunes en el huésped (2). 
Bimstein y Ebersole en 1989, mencionan que Mounton et al. afirman que las reacciones inmunológicas asociadas con enfermedad periodontal han sido identificadas por la aparición de anticuerpos séricos circulantes contra microorganismos en placa subgingival, por la síntesis y deposición de inmunoglobulinas y en algunos casos componentes complementarios en la encía humana y fluido crevicular, como también por la presencia de niveles incrementados de inmunoglobulinas salivares en pacientes con periodontitis (2).

Bimstein y Ebersole en 1989, refieren que el tejido gingival con inflamación crónica en adultos presenta un número similar de linfocitos y de células plasmáticas, además de características de lesión periodontal establecida; en tanto que, los mismos tejidos en niños presentan características de una lesión temprana en la cual los linfocitos T infiltran histológicamente al tejido conectivo y hay poca presencia de neutrófilos. Un incremento en la respuesta de Ig G a medida que aumenta la edad puede ser regulada por un incremento de células T en el infiltrado celular inflamatorio. El aumento de linfocitos $\mathrm{T}$ puede ejercer gran influencia en la expresión clínica de la inflamación, puesto que estas células, tienen la capacidad de producir citoquinas que favorecen la destrucción tisular (2).

Romero et al. en 2011, en estudio realizado sobre la cuantificación de los niveles de inmunoglobulinas A, G y M en saliva de 177 niños entre 3 y 12 años, sanos y con gingivitis, registraron datos en cuanto al índice gingival y de placa bacteriana, encontrando que a medida que el individuo crece aumenta el índice gingival, en presencia de menores cantidades de placa bacteriana. Se concluyó que la edad es un mejor predictor de los niveles de IgA que el índice 
gingival y el índice de placa bacteriana. Estos resultados confirman que definitivamente los niveles de IgA se incrementan con la edad en respuesta al constante reto microbiano que se encuentra en la cavidad oral, sin mostrar relación con la enfermedad gingival; además se encontró que el índice gingival es mejor predictor de los niveles de IgM que la edad y el índice de placa bacteriana (41). Es de resaltar la importancia de las características inmunológicas en la respuesta del tejido periodontal ante la presencia de la biopelícula dental y la severidad de la enfermedad gingival a diferentes edades del individuo.

\section{DISCUSIÓN}

Sobre las características morfológicas, se confirmó la presencia de la papila retrocusídea, una estructura propia del periodonto de niños y jóvenes en la encía insertada; localizada en lingual del canino mandibular a $3 \mathrm{~mm}$ del margen gingival. En una muestra de 300 niños en una población de 3 a 12 años de la ciudad de Bogotá, dicha estructura se presentó en un 78,5 \% de los niños, en la mayoría de los casos presentó ubicación bilateral, forma ovalada, un tamaño de 0-3 mm, consistencia blanda, color rojo y predominantemente de base sésil y aspecto liso (42).

Además, en otros hallazgos de Romero et al. en 2013, sobre diferencias histomorfológicas entre tejido clínicamente sano de niños y adultos jóvenes se corroboran en el periodonto en dentición decidua, la presencia del infiltrado celular inflamatorio del tejido conectivo adyacente al epitelio de unión, además, un área de epitelio de unión mayor en los tejidos periodontales de los niños, mayor grosor y número de capas en la zona apical. También se pudo observar mayor cantidad de vasos sanguíneos, un predominio fibras de tejido contectivo laxas y paraqueratinización del 
epitelio oral. No se encontraron diferencias en la amplitud de los tejidos ni en su espesor con respecto a los epitelios oral y surcular, tampoco en la dimensión de tejido conectivo ni en el grosor de la capa córnea (43).

En cuanto a los factores asociados a la enfermedad gingival y materiales restaurativos en niños; Romero et al. en 2017, llevaron a cabo un estudio clínico sobre el estado gingival de los dientes temporales con y sin restauración de coronas de acero en niños de 3 a 9 años. Los resultados demostraron que no existió una relación entre la adaptación y el estado gingival, como tampoco se observaron diferencias entre la salud gingival de los tejidos periodontales de dientes restaurados con coronas de acero y su antímero no restaurado (44).

En el año 2013 se llevó a cabo una revisión sistemática de la literatura, Arias et al. (45), sobre los criterios diagnósticos y alternativas de tratamiento de periodontitis en población pediátrica. En dicha revisión, ningún artículo cumplió con los criterios de inclusión, principalmente debido a que no cumplieron con el rango de edad establecido y, además, porque no alcanzaron el grado de evidencia requerido por el tipo de estudio encontrado como reportes o series de caso. Este resultado puede deberse a diferentes factores, como la baja incidencia y prevalencia de las patologías estudiadas que no permite llevar a cabo estudios con un diseño de investigación con mayor jerarquía y grado de evidencia que permitan tener mayor tamaño de muestra.

En cuanto a las características microbiológicas, es claro que existe una relación entre la colonización temprana de la microbiota supra y subgingival con la presencia de inflamación gingival en niños de mayor edad, con predominio de patógenos periodontales como T. forsythia, 
P. gingivalis y $T$. denticola. Las especies bacterianas más frecuentes en biopelícula supra y subgingival fueron especies de Actinomyces, V. párvula, S. mitis, S. sanguinis, C. gingivalis, E. corrodens, $F$. nucleatum, $P$. intermedia y $P$. nigrescens (36). La evidencia encontrada sobre la condición sistémica, la condición sociodemográfica, la interacción bacteriana, la composición microbiana y el estado periodontal es limitada y no es concluyente (39).

Respecto de las características inmunológicas, Genco y Slots, Page, Bimstein y Ebersole, citados por Bimstein y Ebersole en 1991, sustentan que la manifestación de la reacción inflamatoria depende de la interacción entre los factores locales y los sistémicos incluyendo la respuesta inmunológica del individuo (3). El infiltrado celular inflamatorio de la encía con gingivitis en niños compromete especialmente linfocitos T y una respuesta humoral con activación de linfocitos B, que sintetizan anticuerpos como las IgA, IgG e IgM. El sistema inmune del niño preescolar es inmaduro y dichas inmunoglobulinas séricas alcanzan sus niveles más altos hacia la pubertad.

De esta manera, estudios de Tolo et al., citados por Bimstein y Ebersole en 1989 (2), indican que los niveles séricos de anticuerpos contra microorganismos involucrados en la enfermedad periodontal se incrementan gradualmente de la infancia a la adultez.

\section{CONCLUSIONES}

Sobre las características morfológicas se puede decir que, en el tejido gingival de los dientes, los productos de la biopelícula se expresan con una respuesta inflamatoria de los mismos, y su 
manifestación depende de varios factores locales y sistémicos, además del período de tiempo en que esté presente la biopelícula (2). De acuerdo con Lang y Bartold (2018), pueden agruparse en tres categorías mayores: microbiológicas, por compuestos de la biopelícula supra y subgingival; del medio ambiente, por factores como nutrición, estrés, medicaciones y tabaquismo; y del huésped, determinado por factores predisponentes locales como bolsas periodontales, restauraciones dentales y anatomía radicular; y por factores modificantes sistémicos como la función inmune, la salud sistémica y la genética (1).

En los niños de los diferentes grupos etarios, se concluyó que la severidad de la gingivitis se presentó de forma incipiente en un $90 \%$ y no hubo presencia de gingivitis severa (18).

Sobre los criterios diagnósticos y alternativas de tratamiento de periodontitis en población pediátrica, las estrategias de promoción y prevención de la enfermedad periodontal deben basarse en un diagnóstico claro, que permita proponer correctamente un plan de tratamiento. Sin embargo, existe poca literatura que reporte con evidencia sobre el diagnóstico periodontal en niños y las alternativas de tratamiento de las enfermedades periodontales destructivas con tasa de progresión rápida o como manifestación de enfermedades sistémicas según la actual clasificación.

Sobre las características microbiológicas no existe suficiente evidencia científica que sustente la relación entre la composición bacteriana con el estado gingival y periodontal en niños de tres a doce años. No obstante, se sugiere que a medida que se incrementa la edad se favorece la presencia de patógenos periodontales relacionados con la presencia de enfermedad periodontal (39). 
Sobre las características inmunológicas, uno de los aspectos interesantes del desarrollo de la enfermedad gingival en niños es la menor severidad de la respuesta clínica frente a similares parámetros desencadenantes como puede ser la biopelícula dental, comparado con lo que se observa en los adultos. Una posible explicación de esta diferencia puede ser el comportamiento de la respuesta inmune que madura en la medida en que el individuo se enfrenta al reto antigénico hasta alcanzar límites máximos en la edad adulta (41).

Como conclusión general, en concordancia con Matsson en 1993, durante ciertos períodos, el riesgo a desarrollar gingivitis es muy bajo, mientras que en otros, los tejidos gingivales son más susceptibles a reaccionar con un proceso inflamatorio a la irritación bacteriana; de esta manera, se debe apuntar que ni la cantidad de biopelícula ni un cambio repentino en su composición, excepto por los cambios relacionados con la edad, podrían explicar la reacción gingival relativamente leve en niños y adolescentes jóvenes (9).

\section{REFERENCIAS}

1. Lang NP, Bartold PM. Periodontal health. J Clin Periodontol. 2018 Jun; 45 20: S9-S16. https://doi.org/10.1111/jcpe.12936

2. Bimstein, E, Ebersole J. The age-dependent reaction of the periodontal tissues to dental plaque. ASDC J Dent Child. 1989; 56(5): 358-362.

3. Bimstein E, Ebersole JL. Serum antibody levels to oral microorganisms in children and young adults with relation to the severity of gingival disease. Pediatr Dent. 1991; 13(5): 267-272.

4. Matsson L, Goldberg P. Gingival inflammatory reaction in children at different ages. J Clin Periodontol. 1985; 12(2): 98-103. https://doi.org/10.1111/j.1600-051x.1985.tb01368.x 
5. Matsson L. Development of gingivitis in pre-school children and young adults. A comparative experimental study. J Clin Periodontol. 1978; 5(1): 24-34. https://doi.org/10.1111/j.1600051x.1978.tb01903.x

6. Peretz B, Machtei EM, Bimstein E. Periodontal status in childhood and early adolescence: three-year follow up. J Clin Pediatr Dent. 1996 Spring; 20(3): 229-232.

7. Drummond BK, Brosnan MG, Leichter JW. Management of periodontal health in children: pediatric dentistry and periodontology interface. Periodontol 2000. 2017; 74(1): 158-167. https://doi.org/10.1111/prd.12195

8. Hugoson A, Koch G, Rylander H. Prevalence and distribution of gingivitis-periodontitis in children and adolescents. Epidemiological data as a base for risk group selection. Swed Dent J. 1981; 5(3): 91-103.

9. Bimstein E, Matsson L. Growth and development considerations in the diagnosis of gingivitis and periodontitis in children. Pediatr Dent. 1999; 21(3): 186-191.

10. Matsson L. Factors influencing the susceptibility to gingivitis during childhood-a review. Int J Paediatr Dent. 1993; 3(3): 119-127. https://doi.org/10.1111/j.1365-263x.1993.tb00067.x

11. Bimstein E. Periodontal considerations in the child dental patient. Acta Odontol Pediatr. 1987; 8(1): 13-19.

12. Delaney J. Periodontal and soft-tissue abnormalities. Dent Clin North Am 1995; 39(4): 837850.

13. Zappler S. Periodontal disease in children. J Am Med Womens Assoc. 195; 6(8): 297-299.

14. Bimstein E. Histologic characteristics of the gingiva associated with the primary and permanent teeth of children. Pediatr Dent.1994; 16(3): 206-210. 
15. Mackler SB, Crawford JJ. Plaque development and gingivitis in the primary dentition. J Periodontol 1973 Jan; 44(1): 18-24. http://doi.org/10.1902/jop.1973.44.1.18

16. Arango JE, Salgado JF, Tobón M. Prevalencia de gingivitis en niños de 5 a 8 años. CES Odontol. 1993; 6(1).

17. Taboada O, Talavera I. Prevalencia de gingivitis en una población preescolar del oriente de la ciudad de México. Bol Med Hosp Infant Mex. 2011; 68(1): 21-25.

18. Zaror Sánchez C, Muñoz Millán P, Sanhueza Campos A. Prevalencia de gingivitis y factores asociados en niños chilenos de cuatro años. Av Odontoestomatol. 2012; 28 (1): 33-38.

19. Benítez P. Romero MR. Gingivitis en la niñez y pubertad temprana. (Trabajo de grado para optar al título de odontólogo). Bogotá, Colombia: Pontificia Universidad Javeriana; 2002.

20. Baer P, Benjamin SD. Pathology of periodontal disease in children: development of the gingival inflammatory lesion. In: Baer PN, Benjamin SD (eds). Periodontal disease in children and adolescents. Philadelphia, PA: Lippincott; 1974. pp. 17-32.

21. Longhurst P, Johnson NW, Hopps RM. Differences in lymphocyte and plasma cell densities in inflamed gingiva from adults and young children. J Periodontol. 1977; 48(11): 705710. http://doi.org/10.1902/jop.1977.48.11.705

22. Walker JDE. Periodontal disease in children and adolescents. In: Stewart RE, Barber TK, Troutman KC, Wei SHY (eds). Pediatric dentistry scientific foundations and clinical practice. St Louis, MO: Mosby; 1982. pp. 623-639.

23. Bimstein E, Soskolne WA, Lustmann J, Gazit D, Bab I. Gingivitis in the human deciduous dentition. J Clin Periodontol 1988; 15(9): 575-580. http://doi.org/10.1111/j.1600$\underline{051 x .1988 . t b 02132 . x}$ 
24. Romero MR, Colmenares MM, Benitez AP, Baltodano FE, Pacheco BJ. Diferencias histológicas en la encía de niños y adultos jóvenes con gingivitis inducida por biopelícula. Univ Odontol. 2011; 30(65): 79-88.

25. Jimenez M. The normal periodontium in children. Cent Estud Recur Odontol Niño. 1983; 8(1): $5-23$.

26. Durr DP, Ashrafi MH, Duncan WK. A study of plaque accumulation and gingival health surrounding stainless steel crowns. ASDC J Dent Child. 1982; 49(5): 343-346.

27. Henderson HZ. Evaluation of the preformed stainless-steel crown. ASDC J Dent Child. 1973; 40(5): 353-358.

28. Myers DR. A Clinical study of the response of the gingival tissue surrounding stainless steel crowns. J Dent Child. 1975; 42(4): 281-284.

29. Ercoli C, Caton JG. Dental prostheses, and tooth-related factors. J Clin Periodontol. 2018; 45(Suppl 20): S207-S218. http://doi.org/10.1111/jcpe.12950

30. Madrigal D, Viteri EM, Romero MR, Colmenares MM, Suarez A. Factores predisponentes para la inflamación gingival asociada a coronas de acero en dientes temporales en la población pediátrica. Rev Fac Odontol Univ Antioq. 2014; 26(1): 152-163.

31. Checchio LM, Gaskill WF, Carrel R. The relationship between periodontal disease and stainless-steel crowns. ASDC J Dent Child. 1983; 50(3): 205-209.

32. Ridgeway EE. Periodontal disease: diagnosis and management. J Am Acad Nurse Pract. 2000; 12(3): 79-84. http://doi.org/10.1111/j.1745-7599.2000.tb00171.x

33. Papapanou PN, Sanz M, Buduneli N, Dietrich T, Feres M, Fine DH, Flemmig TF, Garcia R, Giannobile WV, Graziani F, Greenwell H, Herrera D, Kao RT, Kebschull M, Kinane DF, Kirkwood KL, Kocher T, Kornman K S, Kumar PS, Loos BG, Tonetti MS. Periodontitis: 
Consensus report of Workgroup 2 of the 2017 World Workshop on the Classification of Periodontal and Peri-Implant Diseases and Conditions. J Clin Periodontol. 2018; 89(Suppl 1): S173-S182. http://doi.org/10.1002/JPER.17-0721

34. Albandar JM, Susin C, Hughes FJ. Manifestations of systemic diseases and conditions that affect the periodontal attachment apparatus: Case definitions and diagnostic considerations. $\mathrm{J}$ Clin Periodontol. 2018; 45(20): S171-S189. http://doi.org/10.1111/jcpe.12947

35. Papaioannou W, Gizani S, Haffajee AD, Quirynen M, Mamai-Homata E, Papagiannoulis L. The microbiota on different oral surfaces in healthy children. Oral Microbiol Immunol. 2009; 24(3): 183-189. http://doi.org/10.1111/j.1399-302X.2008.00493.x

36. Kamma JJ, Diamanti-Kipioti A, Nakou M, Mitsis FJ. Profile of subgingival microbiota in children with primary dentition. J Periodontal Res. 2000; 35(1): 33-41. http://doi.org/10.1034/j.1600-0765.2000.035001033.x

37. Darby I, Curtis M. Microbiology of periodontal disease in children and young adults. Periodontol 2000. 2001; 26: 33-53. http://doi.org/10.1034/j.1600-0757.2001.2260103.x

38. Gafan GP, Lucas VS, Roberts GJ, Petrie A. Wilson M, Spratt DA. Prevalence of periodontal pathogens in dental plaque of children. J Clin Microbiol. 2004; 42(9): 4141-4146. http://doi.org/10.1128/JCM.42.9.4141-4146.2004

39. Querubín MC, Vela YS, Romero MR, Colmenares MM, García DA, Suárez A. Estado periodontal y microbiota del surco gingival en dentición temporal y mixta. Revisión sistemática. Univ Odontol 2020.

40. Drummond B, Brosnan MG, Leichter JW. Management of periodontal health in children: pediatric dentistry and periodontology interface. Periodontol 2000. 2017 Jun; 74(1): 158-167. https://doi.org/10.1111/prd.12195 
41. Romero MR, Lozano ML Posada C, Rueda PA, Roa N, Rodríguez A. Immunoglobulin A, G and $\mathrm{M}$ levels in saliva in children between 3-12 years of age, healthy and with gingivitis. Acta Odontol Latinoam. 2011; 24 (2): 176-182.

42. Ramírez L, Ramos G, Romero M. Colmenares M. Presencia de la papila retrocuspídea en una población infantil de 3 a 12 años de Bogotá. Univ Odontol. 2002; 22(50): 9-14.

43. Romero MR, Colmenares M, Arévalo C, Vargas A. Diferencias histomorfológicas entre tejido gingival clínicamente sano de niños y adultos jóvenes. Acta Odontol Venez. 2013; 51(2).

44. Romero MR, Colmenares MM, Valle MV, Andrade MB, Suarez A, Estado gingival de dientes temporales con y sin restauración de coronas de acero en niños de 3 a 9 años. Univ Odontol. 2017;36(77). https://doi.org/10.11144/Javeriana.uo36-77.egdt

45. Arias DC, Herrera LX, Romero MR, Velosa J. Diagnóstico y alternativas de tratamiento de las enfermedades periodontales agresivas o como manifestación de enfermedad sistémica en niños entre 3 y 12 años de edad. Revisión sistemática de la literatura. Univ Odontol 2013; 32(69): 123-146. 